\title{
FRAMEWORK PROPOSAL FOR A BIM-BASED DIGITAL PLATFORM FOR FLEXIBLE DESIGN AND OPTIMIZATION OF INDUSTRIAL BUILDINGS FOR INDUSTRY 4.0
}

\author{
Julia Reisinger ${ }^{1}$, Iva Kovacic ${ }^{2}$, Hannes Kaufmann ${ }^{3}$, Peter Kán ${ }^{4}$, Iana Podkosova ${ }^{5}$
}

\begin{abstract}
The production of the future, respectively industry 4.0, is a highly networked, digitized and thus individualized production, leading to shortened product lifecycles and constantly changing processes. Industrial buildings need to be capable to react to these varying conditions, making the realization of flexible building systems necessary. Inflexible building structures lead to early rescheduling or even demolishing, resulting in increased life cycle costs and material demand. The load-bearing structure is decisive for maximum flexibility as it is the most rigid element with the longest service life. The planning of flexible building structures and the consideration of production in building design requires maximum integration of all stakeholders in early design stage. However, early integration of all stakeholders, processes and tools is rare and difficult due to the lack of interoperability of domain-specific software and sequential planning methodology. This paper presents the ongoing research conducted within the research project BIMFlexi. The goal of BIMFlexi is to make industrial buildings efficiently adaptable to rapidly changing production processes by developing an integrated BIM-based digital platform to enable flexible structural analysis, taking into account changing production processes and support in multi-objective optimization and decision support. In this paper, potentials and limits for integrating processes and discipline specific models of building and production planning are identified and a framework for a "BIM-based digital Platform for Flexible Design and Optimization of Industrial Buildings for Industry 4.0" proposed. The proposed framework couples digital tools such as BIM, parametric modelling, structural analysis and VR within a platform to allow multi-objective optimization and early decision making in real-time.
\end{abstract}

Keywords: BIM-based digital platform, integrated planning, industrial building, multi-objective optimization, performance-based structural design.

1 Univ. Ass. PhD Student, Institute of Interdisciplinary Construction Process Management, Vienna University of Technology, 1040 Vienna, Austria, julia.reisinger@tuwien.ac.at

2 Univ.-Professor., Institute of Interdisciplinary Construction Process Management, Vienna University of Technology, 1040 Vienna, Austria, iva.kovacic@tuwien.ac.at

3 Assoc. Professor, Institute of Visual Computing and Human-Centered Technology, Vienna University of Technology, 1040 Vienna, Austria, hannes.kaufmann@tuwien.ac.at

4 PostDoc Researcher, Institute of Visual Computing and Human-Centered Technology, Vienna University of Technology, 1040 Vienna, Austria, peter.kan@tuwien.ac.at

5 PhD Student, Institute of Visual Computing and Human-Centered Technology, Vienna University of Technology, 1040 Vienna, Austria, yana.podkosova@tuwien.ac.at 
Framework proposal for a BIM-based digital platform for flexible design and optimization of industrial buildings for Industry 4.0

\section{INTRODUCTION}

The fourth industrial revolution is already established in many manufacturing industries (Woodhead et al., 2018) but the benefit in terms of improvements in productivity and quality, has not gained much attention in the construction industry (Oesterreich and Teuteberg, 2016). Short production cycles and constantly changing production systems are characterizing the production of the future. For industrial buildings, these rapid changing processes mean extremely short planning and construction times, as well as the necessity of implementing highly flexible building systems. In future, buildings, including structural and energy systems, must be able to react to these production changes, individual customer requirements and changes in use (Wiendahl et al., 2014, Bracht et al., 2018). Decisive for a building's adaptability are the structural and building service equipment (BSE) systems. The ability to convert and retrofit is most strongly influenced by the load-bearing structure, as the most rigid element with the longest service life. Structural engineers are usually involved too late in the traditional planning process, resulting in suboptimal floor plans and load-bearing structures, poor building quality and reduced utilization (Li, 2018). Mostly rescheduling or even demolishing and new construction are the result.

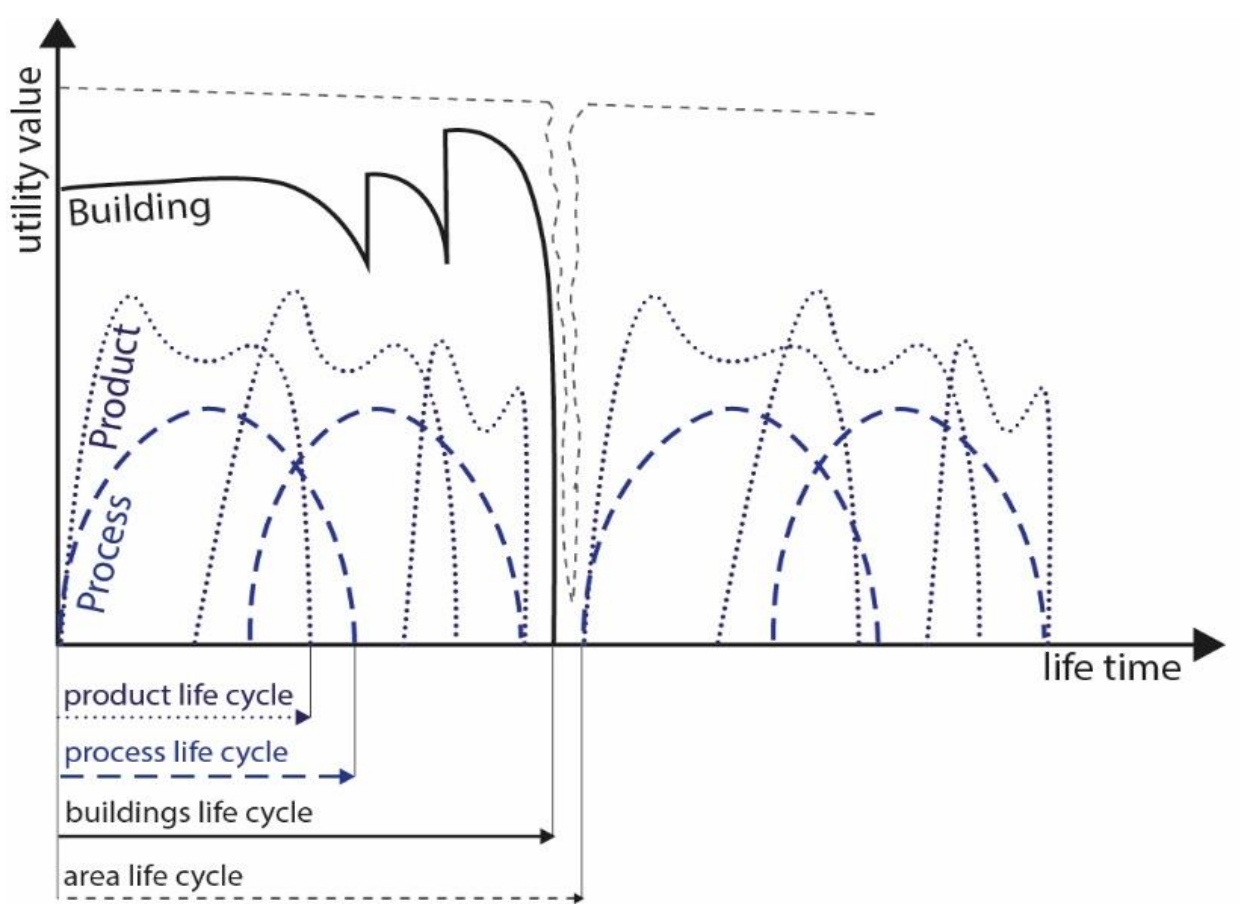

Figure 1: Life cycles of a factory -own presentation based on Wiendahl et al. (2007)

Wiendahl et al. (2007) highlight the importance of designing structures, which are able to meet the changing requirements of production businesses. Integrated planning in industrial construction requires a high degree of networking, coupling and coordination of processes and discipline specific-models for all involved stakeholders, leading to increased complexity. Although digitization and Building Information Modeling (BIM) deployment in the construction industry are advancing rapidly, most common digital tools' configuration is domain-specific for single disciplinary use (silo-use) and offer a low degree of interoperability. Especially in early design stage, which is crucial for the 
definition of essential parameters influencing the entire life cycle, the identification and integration of all stakeholders, processes and tools is rare and, in addition, technically fraught with obstacles due to the lack of interoperability of domain-specific software tools and process-related because of sequential planning methodology. Integrated industrial building models, which integrate production into building design and vice versa, are however, rare and data exchange and data continuity do not exist.

New digital technologies, such as BIM, algorithmic modelling, automation methods or Augmented and Virtual Reality (AR/VR), have the potential to support interdisciplinary industrial construction projects, but are not fully exploited yet. Flexibility and neutrality in use, an integrated planning methodology in early design stage, the interoperability of digital tools and methods for multidisciplinary optimization and decision support were identified as the most important requirements for planning and operation of flexible industrial buildings.

This paper presents the ongoing research, conducted within the research project BIMFlexi, funded by the Austrian Ministry for Transport, Innovation and Technology through the Austrian Research Promotion Agency FFG (Grant No.: 877159). The primary goal of BIMFlexi is to make industrial buildings efficiently adaptable to rapidly changing production processes by integrating production planning into building design. This paper investigates potentials of coupling digital planning methods (BIM, generative design and structural analysis) with multi-objective optimization methods and multi-user VR to support integrated industrial building design. Its main goal is the proposal of a novel framework for a "BIM-based digital Platform for Flexible Industrial Building Design 4.0", enabling the integration of production into building design, supporting design, analysis and optimization of flexible building structures by providing visualization and decision support in real-time.

\section{LITERATURE REVIEW}

\subsection{Flexibility in Structural Design}

The structural system strongly influences the transformability of a production system. The supporting structure must allow changes and be technologically upgradeable with a minimum of capital and technological investment. If the load-bearing structure is designed for flexibility, costs and time required for renovation can be reduced (Slaughter, 2001). The rigid supporting structure has a service life of approximately 50 to 100 years; in comparison, the BSE has a service life of 10 to 20 years (Fastabend, 2002). Flexible load-bearing structures, which can be implemented by means of wide-span ceiling or girder systems, sufficiently high storeys and different load carrying capacities, can prolong building's service life without expensive conversion measures (Graubner, 2014). Early variant studies taking into account possible scenarios of production layouts could support in designing flexible and re-configurable load bearing structures. However, early variant studies considering multi-criteria parameters are rarely carried out in structural design, as they are complex and require maximum stakeholder integration.

\subsection{Integrated Planning Methodology in Industrial Building Design}

AEC belongs to the least digitized industries, still caught in silo-thinking and sequential planning processes (Schober, 2016, Agarwal, 2016). As an emerging digital tool, BIM offers a common digital knowledge platform integrating the activities of all stakeholders along the construction value chain (Succar, 2009, Zhang et al., 2018). BIM aims at the 
integration of different data models - with geometric and/or non-geometric (e.g. costs, time, physical properties, etc.) information - as well as data generation, exchange and processing in interdisciplinary processes (Sibenik and Kovacic, 2018). BIM and computer-aided simulations are already used in isolated cases, but there is still a lack of interoperability and data consistency. The applications are still in classical domainspecific thinking and silo attitude of the data of the different planning disciplines, resulting in information and data losses (Rahmani Asl et al., 2015). However, the goal of seamless global software interoperability is far from being achieved in AEC industry (Grilo and Jardim-Goncalves, 2010). The discrepancy between product-dependent interfaces and the resulting incomplete transfers of geometry and attributes prevent error-free data and information transfer. IFC (Industry Foundation Classes) is the most widely used scheme for integrated planning. IFC-based exchange is currently the most used standard for BIM-based data exchange, with over 150 software tools supporting the scheme (buildingSMART, 2018). The data exchange workflow using IFC building data is still sequential. However, this integrated scheme as a data management concept in AEC industry does not take into account production planning.

\subsection{BIM in Industrial Construction Projects}

Woodhead et al. (Woodhead et al., 2018) see data integration as the key factor for value creation and a need to overcome the tendency to use point solutions in construction industry. Even if there are approaches and concepts for a digital factory, holistic methods and tools, integrating production processes, machine layout and building and BSEplanning are still lacking. Since industrial construction projects are involving multidisciplinary teams and processes, the application of BIM offers a great opportunity to support integrated production and building planning (Oesterreich and Teuteberg, 2016).

However, the integration of production systems into building design possess a great challenge for BIM processes and tools (Näser and Wickenhagen, 2018). BIM application and workflows within manufacturing companies, the interfaces and the data exchange with other related departments in an industrial company have been little researched (Ma et al., 2017). An integrated model in industrial building design, which links production with building design, is recommended, but is not yet available (Kampker et al., 2013, Büscher et al., 2014). Wiendahl et al. (2014) propose the approach of "synergetic factory planning", integrating the two disciplines on process level and without using BIM. Näser and Wickenhagen (2018) investigate the integration of factory planning into building design with BIM, from production system planning perspective. Bracht et al. (2015) examine the collaboration in a virtual production project space with the aim of faster elimination of collisions, promotion of continuous communication and improvement of planning quality. The integration of the production system planning into the building design, from production planner's perspective and for the operation phase (post-planning) is conducted at TU Dortmund (Lenz et al., 2019, Delbrügger et al., 2017).

\subsection{Computational Tools for Structural Analysis}

Current tendencies in structural design point towards an increased use of BIM and parametric design tools embedded in Rhinoceros 3D platform (Associates;, 2020b) such as Grasshopper (Associates; 2020a), Karamba3D (Preisinger, 2020) etc. next to the common analysis tools based on Finite Element Method (FEM). Most FEM-based structural analysis tools are not integrated into the BIM working environment (Hasan et al., 2019). Each analysis software has its own way of defining the analysis model, leading 
to many challenges in implementation of BIM in structural design, such as lack of software interoperability and data exchange problems. Despite the considerable progress in the development of open standards, software tools are still not able to provide a functional and reliable exchange between domain-specific models (Laakso and Kiviniemi, 2012). The most commonly used Open Standard for the exchange of architectural and structural models is IFC (Dib). Sibenik (Sibenik and Kovacic, 2018) evaluated BIM-based structural design and its data exchange and identified that current available certified software tools do not provide sufficient possibilities for a satisfactory data exchange as they do allow interoperability only to a limited extent. The use of BIM in early design stage is a great challenge, as it requires a high degree of explicit knowledge, which is often not available at that time. In addition, structural analysis tools are not efficient for early design stage, as their goals is on precision rather than flexibility (Rolvink et al., 2019). Parametric Modelling requires less explicit knowledge than BIM. Parametric modeling supports in the initial phase of projects, since an early integration of engineering-specific knowledge is possible (Sacks and Barak, 2008) and dynamic geometry and information descriptions by variable parameters allow fast variant studies, enabling a flexible design method and evaluation (Shea et al., 2005). However, standard parametric tools offer little information for multidisciplinary analysis and optimization in simulation-based, engineering-specific software (Pan et al., 2019, Mora et al., 2008). Parametric tools have the potential to couple BIM and structural analysis to carry out early structural performance simulations.

\subsection{Structural Performance and Multi-Objective Optimization}

Simulation-based optimization can quickly support multidisciplinary teams in the exploration of multiple design variants, as parametric design generates numerous design alternatives. However, multi-objective optimizations require maximum integration, while often the necessary interoperability of existing modeling, simulation and visualization tools is missing (Rahmani Asl et al., 2015). In order to design and optimize a structural system a number of important decisions need to be made, relating to other disciplines. However, AEC professionals generate and analyze very few design alternatives in the concept phase because of limitations in processes and software tools (Flager et al., 2009). In structural design, optimization methods are rarely carried out because they are time-consuming and inflexible. As a result, planners make decisions with little or no information about the decision's outcome and impact on the overall performance (Basbagill et al., 2017, Mueller and Ochsendorf, 2015).

Few structural analysis methods allow analysis and visualization in a single environment, provide feedback only to the structural engineer himself and do not support an integrated performance improvement (Mueller and Ochsendorf, 2015). Building geometry and construction are directly dependent on the results of the structural analysis (Schmidt et al., 2010). Current tools and processes do not support a fast generation and evaluation of multi-objective results in a structural design process or the handling is complex and error-prone (Mueller and Ochsendorf, 2015, Brown and Mueller, 2016). Furthermore, the parameter definition for the formulation of integrated evaluation criteria is difficult to assess in early design stage (Chi et al., 2015). Existing multi-objective optimization approaches are often used for customer-specific algorithms or for the optimization of single systems to generate geometric, discipline-specific parameters (Flager et al., 2009, Mueller and Ochsendorf, 2015, Brown and Mueller, 2016, Alexis Danhaive and Mueller, 2015, Gerber et al., 2014). Octopus (Vierlinger, 2019), implemented in Rhino and Grasshopper environment, allows multi-objective 
Framework proposal for a BIM-based digital platform for flexible design and optimization of industrial buildings for Industry 4.0

optimization, searching for many goals at once. The consideration of results and their effects on the overall performance over the whole building service life are often neglected in design processes. (Sanguinetti et al., 2010).

\subsection{Integration of BIM and VR}

The integration of VR into BIM hast the potential to support integrated design with realtime feedback and provide early collision checks. It can interactively simulate buildingrelated scenarios, provide cost and resource estimates, increase communication, improve planning model verification, provide real-time feedback on interactions, and enable integration into early design stage (Rüppel and Schatz, 2011, Lin et al., 2018, Sharma et al., 2017). Numerous research focus on the use of virtual reality to accelerate the design process (Berg and Vance, 2017, Zimmermann, 2008, Whyte et al., 2000).

\subsection{Conclusion}

Building and production planning are currently not linked, so that planning is carried out in two parallel worlds. Integrated planning in industrial construction projects requires a high degree of networking, coupling and coordination of processes and submodels of architecture, structural-, BSE- and production planning. In order to enable integrated modelling and optimization, the early use of digital design tools as well as a continuous data exchange is necessary. Especially in the early design stage, which has the greatest impact on performance in operation phase, flexibility and thus the lifetime of buildings, few holistic methods are available. Current BIM systems use simplified simulation models and lack basic requirements for early multidisciplinary decision support. Occasionally is the focus in multi-objective optimization on the structural performance, resulting in suboptimal building structures and inflexible floor plans. Structural analysis usually refers to numerical analysis, a methodology that hardly allows fast variant generation, evaluation and visualization. The use of VR with BIM is still rare in the design and execution of construction projects.

The research project BIMFlexi addresses the lack of a flexible BIM-based structural design methodology taking into account production planning and investigates appropriate multi-objective optimization methods focusing on the structural performance and visual decision support in real-time.

This paper identifies potentials and limits for integrating processes and sub-models of building and production planning and proposes a framework for a "BIM-based digital Platform for Flexible Design and Optimization of Industrial Buildings for Industry 4.0" coupling BIM, parametric tools and VR to support in early decision making to in longterm increase the buildings' flexibility.

\section{RESEARCH DESIGN - TOWARDS THE FRAMEWORK}

The paper builds up on the interaction of various disciplines, such as architecture, structural engineering and BSE- and production planning. Focus is on the mapping and coupling of respective discipline specific models and data in order to generate a framework for a BIM-based digital platform, which enables the integration of building and production planning and serves as a multi-objective optimization model with decision support. The proposed framework in particular addresses the structural design, as the load-bearing structure is the main obstacle to free production planning and flexibility. 
In order to obtain information about the industrial software eco-systems and discipline-specific digital models, such as BIM-, structural analysis-, production planning- and VR-models, the planning processes of all stakeholders are mapped. This involves checking the individual models on respective characteristics, structure and contents, qualities, composition.

Production planning, architecture, structural and BSE- engineering are directly related, making a combination of the requirements from the as-is analysis necessary. Therefore, possible interface formats of the sub-models and software solutions are analyzed. The conducted mapping enables deriving, aggregating, evaluating and comparing the processes, sub-models, data structures and software tools, defining an integrated industrial building design process 4.0 (see Figure 2).

Succeeding the process mapping and analysis, existing parametric modeling, analysis and optimization tools such as Rhino (Associates; 2020b), Grasshopper (Associates; 2020a), Karamba3D (Preisinger, 2020) and Octopus (Vierlinger, 2019), are comprehensively analyzed on potentials and limits regarding interoperability and coupling ability to BIM and VR. In our future research, the interoperability of this software eco system will be tested and evaluated upon which the most favorable software constellation will be chosen.

Based on the previous process, model and data mapping and analysis the framework proposal for a "BIM-based digital platform for flexible design and optimization of industrial building for Industry 4.0" is generated, presented in the following section.

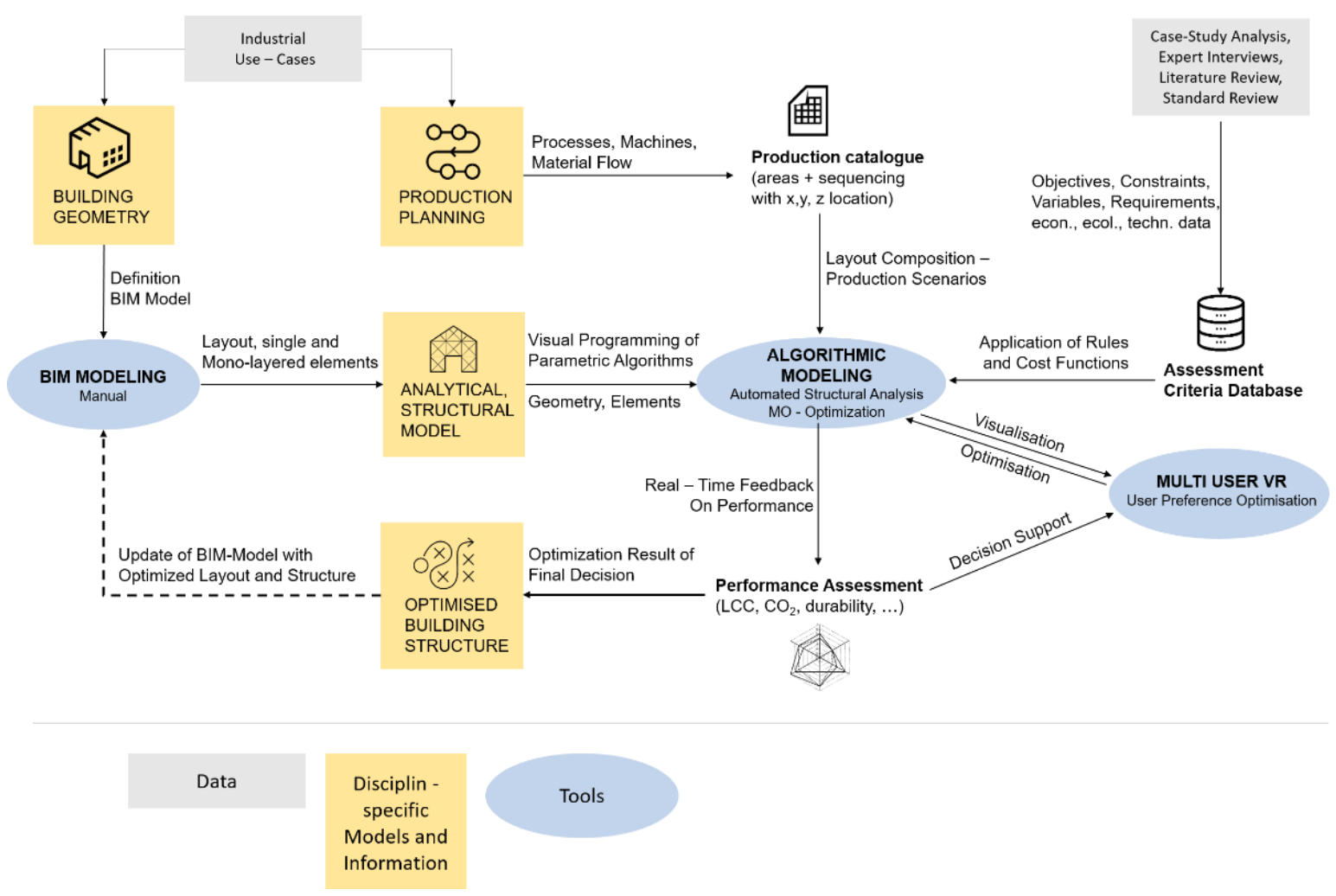

Figure 1: Research Design: Mapping the Processes, Models and Data - Integrated Industrial Building Design 4.0 
Framework proposal for a BIM-based digital platform for flexible design and optimization of industrial buildings for Industry 4.0

\section{FRAMEWORK PROPOSAL}

The main objective of the research project BIMFlexi is to make industrial buildings of industry 4.0 efficiently adaptable to rapidly changing production processes by coupling digital planning methods (BIM, Parametric Modelling and Analysis) with new optimisation algorithms and multi-user VR, taking into account economic, ecological and technical parameters. A framework for an Integrated BIM-based Digital Platform should enable the design, structural analysis and optimization of flexible building structures and serve as visualization and decision support.

The proposed framework approaches the integration of new optimization methods into the BIM-design process, generating multi-objective optimized building structures with minimal user intervention. Furthermore, the integration of multi-user VR into the BIM environment will allow users to quickly and intuitively explore 3D-planning structures and interactively check and manipulate the design to announce their preference for additional optimization. The purpose is not to utilize existing systems and approaches per se; rather, it aims to significantly optimize the design process by integrating different existing tools in one platform.

A consistent data structuring, as basis for model integration, will enable the coupling of software-dependent (BIM software, algorithmic models and VR) and softwareindependent (parameters, requirements and cost functions) data, in a bi-directional data exchange manner, allowing real time feedback. This integration represents one of the major challenges, as the computational runtime should be kept to a minimum in order to enable real time feedback.

Figure 3 displays the proposed framework, which will be tested, evaluated and if necessary modified in further research. The framework consists of six main parts in the process: 1.) BIM-Model, 2.) Algorithmic Script, 3.) Structural Analysis, 4.) Database, 5.) Multi-Objective Optimization, 6.) Multi-User VR Visualization. 


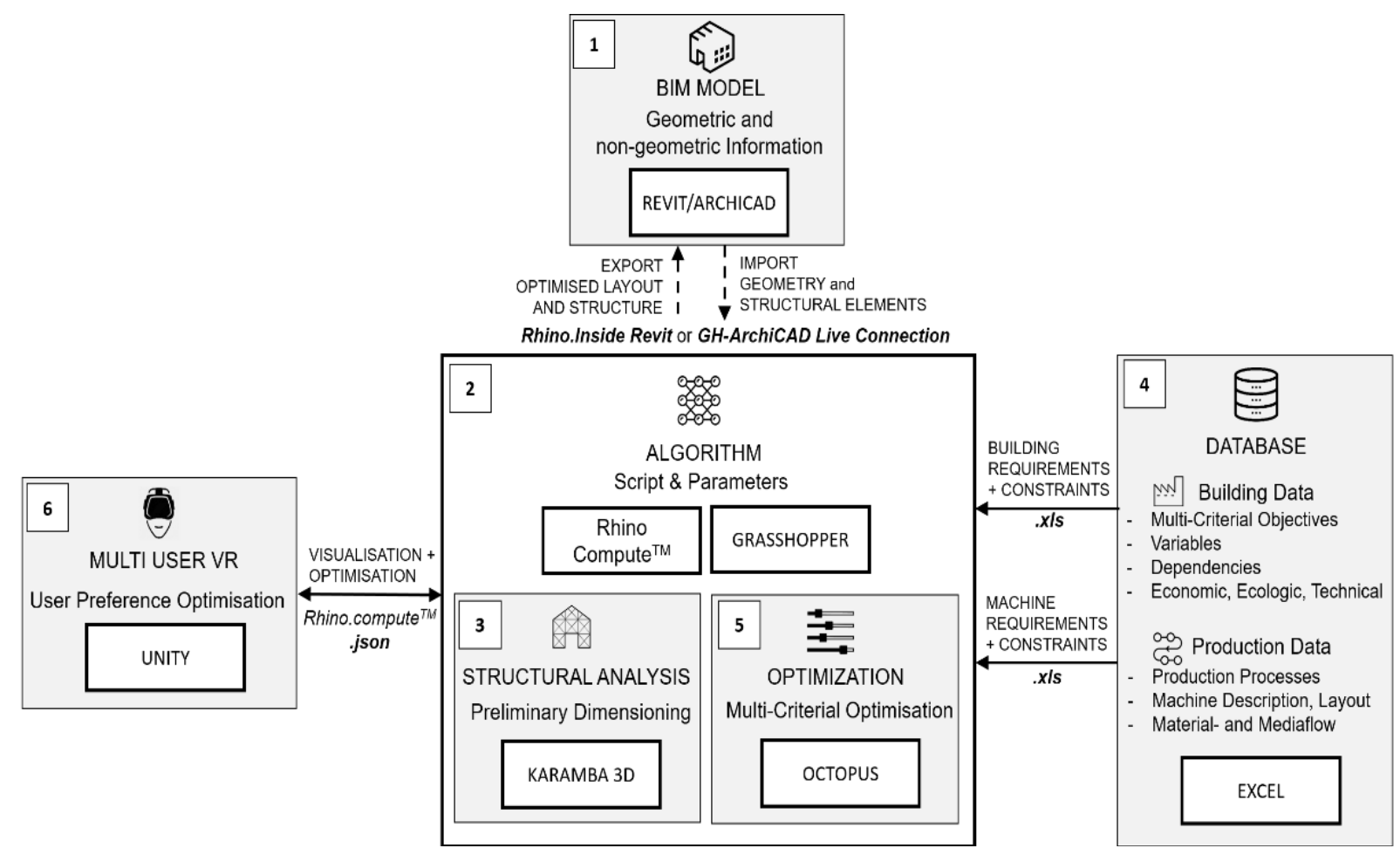

Figure 2: Framework Proposal for a BIM-based digital Platform for Flexible Design and Optimization of Industrial Buildings for Industry 4.0

1.) BIM-Model. A BIM-model of the industrial building will be modeled in a BIM authoring software containing information from architectural design. The BIM software will be tested and evaluated in further research. The BIM-model serves as geometric and non-geometric database. Thereby, the geometry will be pre-defined from the architect in which first assumptions like axis grid system, elements and materials are made. The created BIM-model will be linked to the algorithmic script. The necessary information for seamless data transfer and specific data exchange requirements are prepared by classifying, filtering and pre-processing the geometric and non-geometric data.

2.) Algorithmic Script. For flexible design, making a fast alternative generation for optimization possible, the development of an algorithmic script with linkage to the BIMmodel will be proposed. Within this task, we intend to use Grasshopper as visual programming language. During the research we evaluate whether the existing tools are sufficient or if modifications and own algorithms have to be developed and implemented. From the BIM-model geometry, structural elements and pre-defined necessary data will be imported directly into the algorithmic script. Whereby, data not needed for layout and structural optimization, such as geometric elements from wall or roof cladding, will be excluded from the import. We intend to use Rhino.Inside (Associates;, 2020c) for Revit ${ }^{\circledR}$ (Inc.; 2020) and Grasshopper - ArchiCad Live Connection (SE.; 2020b) for ARCHICAD (SE.; 2020a) and test whether they are suitable for bi-directional data exchange.

3.) Structural Analysis. In order to carry out the structural analysis we propose to extend the algorithmic script using a parametric engineering software e.g. Karam-ba3D, enabling flexible structural design on preliminary design basis. The algorithmic structural analysis script (PARAT) will automatically generate the structural analysis 
model based on geometries and information from the BIM-model. The analysis results will be iteratively used as input for further optimization.

4.) Database. Developing a comprehensive database ensures the collection of all necessary data for Building Design and Production. The building database collects parameters, constraints, objectives and variables for the structural analysis and it contains a definition of the cost function for the multi-objective optimization. Furthermore, it includes ecological, economic and technical parameters for the performance evaluation. The database for production will contain different sets of possible production layouts, defined sets of machines and their requirements to be able to simulate different production process scenarios.

5.) Multi-Objective Optimization. Our proposed multi-objective optimization algorithm will additionally optimize the layout of a building. The variables for layout optimization will mostly contain parameters of load-bearing structures including dimensions and positions of columns, girders, etc. Our optimization will take into consideration the result of structural analysis calculated by Karamba3D. The proposed methodology for structural layout generation is based on the combination of optimization-based and procedural techniques for content generation. The implemented algorithm will iteratively combine the procedural generation of structural elements in a building with the optimization of the layout with respect to objectives of optimization. These objectives will include the following aspects:

- Structural reliability

- Suitability for multiple production plans without the need of changing the building

- Fit with original architectural building layout

- Feedback from decision-makers using the multi-user VR visualization

- Life-cycle costs and material demand

- Other objectives including economical, ecological, technical and other factors

These factors will be combined into a common analytical cost function by scalarization. The cost function will be then optimized in a proposed hybrid optimization process. It is planned to explore the wide solution space of building layouts using genetic algorithms enriched with rule-based procedural generation. The parameters from the planning process, production planning and architectural layout will be used directly as input for the algorithm.

In each iteration of our optimization, several layout proposals will be generated which will be then analyzed in by Karamba 3D. The values from structural analysis will be then taken into account again during the optimization. The BIM-model and PARAT will therefore serve as inputs for the automatic optimization.

Finally, the proposed VR-Exploration in Unity 3D will also serve as a tool for several users to obtain the evaluation of the generated designs. Prior to this step, several proposals will be generated by our optimization and users will be able to specify their preference ratings as well as strict layout requirements in terms of spatial constraints. This user feedback can be taken into account in further optimization process.

6.) Multi-User VR Visualization. The goal of the multi-user VR visualization is to improve the building, resulting from our optimization, by providing the decisions and preferences about suggested layouts and positions of structural elements. These user- 
provided suggestions will be incorporated into further optimization process. Additionally, the proposed multi-user VR visualization will enable decision makers to make the final decision about the planning.

In order for a group of users to be able to evaluate a certain structural layout in VR, a flexible navigation method in virtual space is needed. While simple walking is often considered the most intuitive and effective way of navigation, large spatial dimensions of structural layouts in question make additional navigation methods unavoidable. Such methods supporting collaborating navigation must be developed. New methods will be based on traditional navigation techniques commonly used in VR, including combinations of teleportation and natural walking, however adopted and modified for collaborative use. Using the proposed methodology, the whole group will be able to explore the virtual environment together.

Modifications of the layouts according to user preferences will be accomplished using interaction techniques such as selection and manipulation. VR interaction encompasses a large number of methods, of which the most suitable ones will be chosen (such as ray casting with VR controllers to select objects).

The modifications in the layout performed by users will be translated into additional parameters and constrains that will be fed into the optimization process. Several layouts will be suggested to the group of users in order to select and evaluate the most suitable layout. Finally, decision makers will be able to select the best layout for their needs, the latter being stored and exported as the final solution back into the BIM model.

We intend to use existing tools and evaluate whether they are suitable and sufficient. Furthermore, we will test if the proposed coupling allows a bi-directional data transfer with minimal user invention in the workflow. The proposed framework will then be modified and, if necessary, own algorithms will be developed and implemented.

\section{EXPECTED IMPACT AND RESULTS}

The proposed framework for an Integrated BIM-based digital platform within the BIMFlexi project will introduce three additional aspects to traditional building and structural design methods, including automated layout proposals, coherence with production and real-time feedback in early design stage for decision-making.

The BIMFlexi research aims to develop a methodology for early cooperation of all stakeholders, like building owner, architecture, structural-, BSE-, and production planner, leading to an improved design process. The integration of do-main-specific processes and tools and the improved interoperability between discipline specific models is expected to minimize information and data losses, enabling a holistic improvement of the production facilities' performance.

The proposed framework is expected to improve building design processes in such a way that they better fit the long-term purpose of industrial buildings. It allows multiobjective layout and structural design optimisation, which will in-crease the building's service-life, leading to a reduction in life-cycle costs and resource consumption. Thus, the "BIM-based digital Platform for Flexible Design and Optimization of Industrial Buildings for Industry 4.0" provides useful assistance in interdisciplinary working environments. 
Framework proposal for a BIM-based digital platform for flexible design and optimization of industrial buildings for Industry 4.0

\section{CONCLUSION AND FUTURE OUTLOOK}

The AEC industry is slightly hesitant to implement new technologies and innovations, digitizing its value chains and processes more slowly than other industries. The perceived difficulties to digitization and thus the possibility of establishing a new culture of collaboration and exchange of digital information are mostly costs, sequential planning processes and inconsistent interfaces in software interoperability. Due to missing production system knowledge in AEC domain, digital design workflows lack integration, and existing digital design tools rarely meet the stakeholders' needs. Concluding, there is very little research of integrating production planning with BIM supported building design.

Since $80 \%$ of the life cycle costs of a building occur in the operation phase and $75 \%$ are determined in the design phase, the research project BIM Flexi ad-dresses the early design stage. In this stage, the important parameters for building performance are set and an investigation of diverse design alternatives is crucial. Several methods compose design optimizations to support integrated and performance driven design. The proposed framework of an integrated BIM-based digital platform, presented in this paper, combines BIM, parametric modelling, structural performance and VR.

In the next step of the research, a comprehensive use-case analysis, whereby real industrial projects represent the use-cases, is carried out. The use-case analysis identifies and collects building- and production-specific data and invest-gates interdependencies. Furthermore, objectives and constraints for integrated industrial building design will be investigated and the requirements and parameters for flexible and adaptable loadbearing structures examined in order to set up the database and formulate the mathematical codes for the cost function.

Based on the results of the use-case analysis and obtained data the algorithmic script for automated optimization and structural analysis will be developed. As presented in the framework, a method for linking the algorithmic script to the BIM-Model will be created. The data continuity, interfaces and suitability of the script will be tested on real industrial use-cases and an evaluation of the used tools and methods will be carried out. As soon as a reliable algorithmic script is defined, the optimization and VR integration will be taken into account.

\section{ACKNOWLEDGMENTS}

The authors would like to acknowledge the support by the Austrian funding institution FFG (Österreichische Forschungsförderungsgesellschaft) for the research project "BIMFlexi" (Grant No. 877159).

\section{REFERENCES}

Agarwal, R. S., M.; Chandrasekaran, S.; (2016) "Imagining construction’s digital future". Available at: https://www.mckinsey.com/industries/capital-projects-andinfrastructure/our-insights/imagining-constructions-digital-future\# [Accessed 28.01.2020].

Alexis Danhaive, R. and C. Mueller (2015). Combining parametric modeling and interactive optimization for high-performance and creative structural design. Future Visions, Symposium 2015 Amsterdam. Amsterdam. Proceedings of the International Association for Shell and Spatial Structures (IASS). 
Associates;, M. (2020a). "Grasshopper." https://www.rhino3d.com/6/new/grasshopper.

Associates;, M. (2020b). "Rhinoceros 3D." https://www.rhino3d.com/.

Associates;, R. M. (2020c). "Rhino.Inside ${ }^{\circledR} . "$ https://www.rhino3d.com/inside.

Basbagill, J. P., F. Flager and M. Lepech (2017). "Measuring the impact of dynamic life cycle performance feedback on conceptual building design." Journal of Cleaner Production 164: 726-735.

Berg, L. P. and J. M. Vance (2017). "Industry use of virtual reality in product design and manufacturing: a survey." Virtual Real. 21(1): 1-17.

Bracht, U., D. Geckler and S. Wenzel (2018). Digitale Fabrik - Methoden und Praxisbeispiele, Springer Vieweg.

Bracht, U., N. T. Gorke and J. Spies (2015). "Close Integration of the Virtual Project Room with Factory DMU of the Factory Layout Planning." ZWF Zeitschrift für wirtschaftlichen Fabrikbetrieb 110(10): 639-642.

Brown, N. C. and C. T. Mueller (2016). "Design for structural and energy performance of long span buildings using geometric multi-objective optimization." Energy and Buildings 127: 748-761.

buildingSMART. (2018). https://www.buildingsmart.org/about/.

Büscher, C., H. Voet, T. Meisen, M. Krunke, K. Kreisköther, A. Kampker, D. Schilberg and S. Jeschke (2014). "Improving Factory Planning by Analyzing Process Dependencies." Procedia CIRP 17: 38-43.

Chi, H.-L., X. Wang and Y. Jiao (2015). "BIM-Enabled Structural Design: Impacts and Future Developments in Structural Modelling, Analysis and Optimisation Processes." Archives of Computational Methods in Engineering 22(1): 135-151.

Delbrügger, T., L. Lenz, D. Losch and J. Rosmann (2017). A navigation framework for digital twins of factories based on building information modeling. 2017 22nd IEEE International Conference on Emerging Technologies and Factory Automation (ETFA): 1-4.

Dib, R. R. J. Z. a. H. N. BIM Interoperability for Structure Analysis. Construction Research Congress 2018: 470-479.

Fastabend, M. S., B; (2002). Modification-capable concrete structures. Beton- und Stahlbetonbau 97. Berlin, Germany, Ernst \& Sohn Num 3: 157-162.

Flager, F., B. Welle, P. Bansal, G. Soremekun and J. Haymaker (2009). Multidisciplinary Process Integration and Design Optimization of a Classroom Building. ITcon Vol. 14, pg. 595-612, https://www.itcon.org/2009/38

Gerber, D., K. Abraham, F. Flager, J. Macedo and M. Lepech (2014). Multi-Attribute Decision-Making and Data Visualization for Multi-Disciplinary Group Building Project Decisions. Engineering Project Organization Conference 2014, CO, USA: 115

Graubner, C.-A. u. a. (2014). „Nachhaltig Bauen mit Beton“. Der Stadtbaustein im DAfStb/BMBF-Verbundforschungsvorhaben

Grilo, A. and R. Jardim-Goncalves (2010). "Value proposition on interoperability of BIM and collaborative working environments." Automation in Construction 19(5): 522530. 
Framework proposal for a BIM-based digital platform for flexible design and optimization of industrial buildings for Industry 4.0

Hasan, A. M. M., A. A. Torky and Y. F. Rashed (2019). "Geometrically accurate structural analysis models in BIM-centered software." Automation in Construction 104: 299-321.

Inc.;, A. (2020). "Autodesk REVIT." Retrieved 31.01.2020, from https://www.autodesk.com/products/revit/overview.

Kampker, A., A. Meckelnborg, P. Burggräf and T. Netz (2013). Factory Planning Scrum: Integrative Factory Planning with Agile Project Management. Proceedings International Conference on Competitive Manufacturing : COMA' 13:345-350

Laakso, M. and A. Kiviniemi (2012). "The IFC Standard - A Review of History, Development, and Standardization." Electronic Journal of Information Technology in Construction 17.

Lenz, L., M. Höpfner, P. Spyridis, K. C. Weist and M. Gralla (2019). BIM Approach for Automatic Decision Support: Case Study Fastening Systems in Factory Adaptation Planning. 2019 European Conference on Computing in Construction: 2-8.

Li, Z. (2018). Research on Structural Design Optimization Methods for Civil Engineering Buildings. 8th International Conference on Social Network, Communication and Education (SNCE 2018).

Lin, Y.-C., Y.-P. Chen, H.-W. Yien, C.-Y. Huang and Y.-C. Su (2018). "Integrated BIM, game engine and VR technologies for healthcare design: A case study in cancer hospital." Advanced Engineering Informatics 36: 130-145.

Ma, K., N. Dawood and M. Kassem (2017). "BIM for manufacturing: a case study demonstrating benefits and workflows and an approach for Enterprise Application Integration (EAI)." 16th International Conference on Construction Applications of Virtual Reality, Hong Kong. 11/12/16 - 13/12/16

Mora, R., C. Bédard and H. Rivard (2008). "A geometric modelling framework for conceptual structural design from early digital architectural models." Advanced Engineering Informatics 22(2): 254-270.

Mueller, C. T. and J. A. Ochsendorf (2015). "Combining structural performance and designer preferences in evolutionary design space exploration." Automation in Construction 52: 70-82.

Näser, P. and N. Wickenhagen (2018). Factory integration into building information modeling. wt Werkstattstechnik online Jahrgang 108 (2018). Düsseldorf, SpringerVDI-Verlag GmbH \& Co. KG. 04-2018: 245-250.

Oesterreich, T. D. and F. Teuteberg (2016). "Understanding the implications of digitisation and automation in the context of Industry 4.0: A triangulation approach and elements of a research agenda for the construction industry." Computers in Industry 83: 121-139.

Pan, W., M. Turrin, C. Louter, S. Sariyildiz and Y. Sun (2019). "Integrating multifunctional space and long-span structure in the early design stage of indoor sports arenas by using parametric modelling and multi-objective optimization." Journal of Building Engineering 22: 464-485.

Preisinger, C. (2020). "Karamba3D." Retrieved 29.01.2020, from https://www.karamba3d.com/.

Rahmani Asl, M., S. Zarrinmehr, M. Bergin and W. Yan (2015). "BPOpt: A framework for BIM-based performance optimization." Energy and Buildings 108: 401-412.

Rolvink, A., J. Breider and J. Coenders (2019). Structural Components - a parametric associative design toolbox for conceptual structural design. Symposium of the International Association for Shell and Spatial Structures. Editorial Universitat Politècnica de València. http://hdl.handle.net/10251/7001. 
Rüppel, U. and K. Schatz (2011). "Designing a BIM-based serious game for fire safety evacuation simulations." Advanced Engineering Informatics 25(4): 600-611.

Sacks, R. and R. Barak (2008). "Impact of three-dimensional parametric modeling of buildings on productivity in structural engineering practice." Automation in Construction 17(4): 439-449.

Sanguinetti, P., M. Bernal, M. El-Khaldi and M. Erwin (2010). Real-time design feedback: coupling performance-knowledge with design iteration for decision-making. Proceedings of the 2010 Spring Simulation Multiconference Article No.: 192: 1-8 https://doi.org/10.1145/1878537.1878737.

Schmidt, R., J. Kiendl, K. U. Bletzinger and R. Wüchner (2010). "Realization of an integrated structural design process: analysis-suitable geometric modelling and isogeometric analysis." Computing and Visualization in Science 13(7): 315-330.

Schober, S. H., P.; (2016) "Digitisation in Construction Industry. Building Europe's road to Construction 4.0. ." Civil Economics, Energy \& Infrastructure Competence Center, 16.

SE.;, G. (2020). "Graphisoft ARCHICAD 23." $\quad$ Retrieved 31.01.2020, from https://www.graphisoft.at/archicad/.

SE.; G. (2020). "Grasshopper - ARCHICAD Live Connection." Retrieved 31.01.2020, from https://www.graphisoft.com/archicad/rhino-grasshopper/.

Sharma, S., A. Sawhney and M. Arif (2017). "Parametric Modelling for Designing Offsite Construction." Procedia Engineering 196: 1114-1121.

Shea, K., R. Aish and M. Gourtovaia (2005). "Towards integrated performance-driven generative design tools." Automation in Construction 14(2): 253-264.

Sibenik, G. and I. Kovacic (2018). Proposal for a discipline-specific open exchange framework. 2018 Proceedings of the 35th ISARC, Berlin: 570-577

Slaughter, E. S. (2001). "Design strategies to increase building flexibility." Building Research \& Information 29(3): 208-217.

Succar, B. (2009). "Building information modelling framework: A research and delivery foundation for industry stakeholders." Automation in Construction 18(3): 357-375.

Vierlinger, R. (2019). "OCTOPUS." Retrieved 29.01.2020, from https://www.food4rhino.com/app/octopus.

Whyte, J., N. Bouchlaghem, A. Thorpe and R. McCaffer (2000). "From CAD to Virtual reality: Modelling approaches, data exchange and interactive $3 \mathrm{~d}$ building design tools." Automation in Construction 10: 43-55.

Wiendahl, H. P., H. A. ElMaraghy, P. Nyhuis, M. F. Zäh, H. H. Wiendahl, N. Duffie and M. Brieke (2007). "Changeable Manufacturing - Classification, Design and Operation." CIRP Annals 56(2): 783-809.

Wiendahl, P., J. Reichardt and P. Nyhuis (2014). Handbuch Fabrikplanung: Konzept, Gestaltung und Umsetzung wandlungsfähiger Produktionsstätten. Hanser.

Woodhead, R., P. Stephenson and D. Morrey (2018). "Digital construction: From point solutions to IoT ecosystem." Automation in Construction 93: 35-46.

Zhang, X., S. Azhar, A. Nadeem and M. Khalfan (2018). "Using Building Information Modelling to achieve Lean principles by improving efficiency of work teams." International Journal of Construction Management 18: 300.

Zimmermann, P. (2008). Virtual Reality Aided Design. A survey of the use of VR in automotive industry. Product Engineering: 277-296. 